\title{
Stand und Steuerung der Digitalisierung
}

\begin{abstract}
Viele Unternehmen haben mit der Digitalisierung einhergehende Veränderungen in ihre Strategie integriert. Diese Analyse zeigt, welche Bereiche sich in der Transformation befinden - und dass nur das Controlling, als Steuerungsinstrument des Top-Managements, die nötige Transparenz erzeugen kann, um zu einer konsistenten und holistischen Steuerung der Digitalisierungsbemühungen zu gelangen.
\end{abstract}

\section{Kai Kruk, Tennyson Rajenthiran}

Die digitale Transformation ist nicht nur von strategischer, sondern auch von operativer Relevanz, die COVID-19-Pandemie zeigt das besonders deutlich. Damit Unternehmen künftig resilienter mit neuen Situationen umgehen können, ist es von zentraler Bedeutung, dass sie Digitalisierung als Querschnittsthema verstehen, das über alle Unternehmens-

\section{Zusammenfassung}

- Die Digitalisierung führt in den 30 DAX-Unternehmen zu konkreten Maßnahmen, im Bereich Entwicklung neuer Geschäftsmodelle gibt es jedoch Schwierigkeiten, digitale Aktivitäten umzusetzen.

- Mittels zusätzlicher Steuerungskennzahlen sollte die Unternehmenssteuerung Digitalisierung verbindlich und als messbares Element im Controlling integrieren und so eine umfassende Transparenz und Steuerbarkeit der Digitalisierungsbemühungen herstellen.

- Unternehmen sollten ihr Reporting an die digitale Entwicklung anpassen und die qualitative Zielerreichung sowie Ergebnis- und Fixkostenbeitrag entsprechender Maßnahmen regelmäßig berichten. funktionen hinweg relevant ist und umgesetzt wird. Zwar hat fast jedes DAX-Unternehmen inzwischen einen Chief Digital Officer und eine entsprechende Abteilung etabliert. Welche Funktionen und Bereiche - wie beispielsweise das Controlling - wie und in welchem Maße mit digitalen Maßnahmen befasst sind, ist oftmals jedoch nicht transparent. Diese Analyse der Geschäftsberichte und aktuellen Online-Auftritte der 30 DAX-Unternehmen hat dies nun empirisch untersucht und liefert Hinweise, an welchen Stellen Unternehmenssteuerung und Controlling ansetzen können, um digitale Aktivitäten in allen Bereichen voranzutreiben.

\section{Analyse von Geschäftsberichten der DAX-Konzerne}

Im Rahmen der quantitativen Textanalyse wurden die Geschäftsberichte der DAX-Konzerne explizit nach Begriffen wie Digitalisierung, Transformation oder Vernetzung durchsucht und das Verhältnis zwischen der reinen Nennung von Schlagworten zu konkret durchgeführten Digitalisierungsmaßnahmen analysiert (vergleiche Kasten Digitalisierungsindex). Die Ergebnisse zeigen die aktuellen Digitalisierungsbemühungen der Konzerne und legen nahe, welche Unternehmensfunktionen in dieser Hinsicht konkrete Maßnahmen implementiert haben. 


\section{Digitalisierungsaktivitäten nach Bereichen}

Die Analyse legt offen, dass die meisten Digitalisierungsaktivitäten der 30 DAX-Konzerne in den Bereich der organisatorischen Transformation fallen. Die Konzerne fokussieren sich hier auf interne Aktivitäten wie zum Beispiel die Verschlankung

\section{„Die meisten Digitalisierungsaktivitäten der 30 DAX-Konzerne fallen in den Bereich der organisatorischen Transformation."}

der Organisationsstruktur oder den Aufbau von digitalen Anlaufstellen wie „Hubs“, „Labs“ oder Kompetenz-Centers. Die DAX-Unternehmen setzen in diesem Zusammenhang auch auf externe Kooperationsaktivitäten mit Start-ups, um ihre Innovationskraft zu steigern. Starke Aufmerksamkeit erhält das Thema Digitalisierung auch in den Bereichen Geschäftsmodell, Prozesse und IT-Infrastruktur. Digitale Maßnahmen in Personal-Management, Führung/Steuerung und Kundenerlebnis hingegen sind unterdurchschnittlich repräsentiert.

In der Analyse der Geschäftsberichte nimmt im Durchschnitt aller betrachteten Dimensionen der quantitative Teil des Digitalisierungsindex, also die reine Nennung von Schlagworten, 30 Prozent ein, der qualitative Teil des Digitalisierungsindex hingegen - die Umsetzung konkreter Digitalisie- rungsaktivitäten - insgesamt 70 Prozent. Während der qualitative Teil in allen Bereichen bei Weitem überwiegt, ist die Verteilung im Bereich Geschäftsmodell ausgewogen (vergleiche Abbildung 1). Die Chancen und Potenziale der Digitalisierung auf das aktuelle Geschäftsmodell werden demnach zwar erkannt, konkrete Maßnahmen umzusetzen oder entsprechende digitale Produkte oder Dienstleistungen zu entwickeln, scheint für die Unternehmen jedoch herausfordernd zu sein. Die übrigen fünf Bereiche Kundenerlebnis, Prozesse und IT-Infrastruktur, Personal-Management, Führung/Steuerung sowie organisatorische Transformation weisen eine größere Differenz zwischen qualitativem und quantitativem Teil auf, was für eine konsequentere Transformation spricht.

\section{„Konkrete Maßnahmen umzusetzen oder entsprechende digitale Produkte oder Dienstleistungen zu entwickeln, scheint für Unternehmen herausfordernd zu sein."}

\section{Digitalisierungsaktivitäten nach Branchen}

Der branchenübergreifende Vergleich zeigt, wie unterschiedlich stark die digitale Transformation in den 30 DAX-Konzernen vorangeschritten ist: Während die Branchen Energie und Rohstoffe (ERM), Transport und Logistik (T\&L), Banken, Versicherungen und Immobilien (BIR) sowie Chemie, Phar-

\section{Abb. 1 Verteilung des Digitalisierungsindex im DAX}

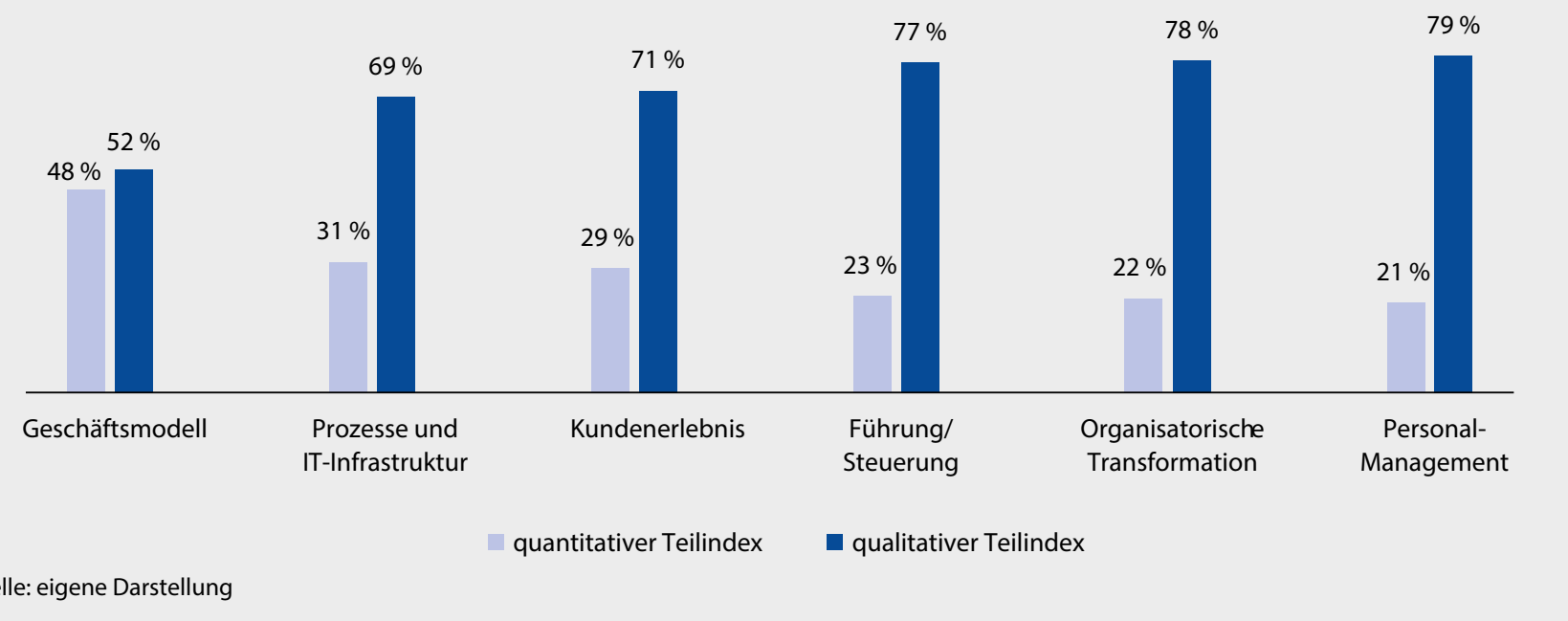




\section{Digitalisierungsindex}

Der Digitalisierungsindex (DI) für jedes Unternehmen wird mittels einer eigens entwickelten Nutzwertanalyse (Scoring-Modell) errechnet, die zwischen der bloßen Nennung von Stichworten (zum Beispiel digital), dem quantitativen Teil, und konkreten Umsetzungsmaßnahmen, dem qualitativen Teil, unterscheidet. Während der quantitative Teil lediglich die Häufigkeit entsprechender Schlagworte ausdrückt, spiegelt der qualitative Teil die konkrete Umsetzung entsprechender Maßnahmen. Der Index ermöglicht es, die Intensität der Digitalisierungsaktivitäten branchenübergreifend zu vergleichen. Der Begriff Digitalisierung wird hierbei ganzheitlich verstanden, das heißt, die Bereiche Geschäftsmodell, Kundenerlebnis, organisationale Transformation, Personal-Management, Führung/ Steuerung, Prozesse und IT-Infrastruktur werden berücksichtigt. Um die Geschäftsberichte systematisch auf digitale Aktivitäten zu untersuchen, wurde das qualitative Inhaltsanalyse-Framework von Mayring (2015) verwendet. Dieser Ansatz wurde durch eine Stichwortsuche ergänzt, um Inhalte und Textstellen mit Bezug zur Digitalisierung zu identifizieren.

ma und Medizin (CPM) einen ähnlichen Indexanteil aufweisen, ist sowohl in der IT-Dienstleistung und Software (ITSS) als auch im Hardware- und Technologiesektor (H\&T) ein höherer quantitativer Anteil im Vergleich zum Durchschnitt zu beobachten. Möglich ist einerseits, dass die beiden genannten

\section{„Unternehmen der ITSS-Branche sind traditionell stärker von der Digitalisie- rung betroffen und bieten viele digitale Lösungen bereits an.}

Branchen in der digitalen Transformation weiter fortgeschritten sind, sodass weniger Digitalisierungsaktivitäten durchgeführt werden. Andererseits könnten auch branchenspezifische Merkmale der Grund sein: So sind beispielsweise Unternehmen der ITSS-Branche aufgrund ihres Geschäftsmodells traditionell stärker von der Digitalisierung betroffen und bieten viele digitale Lösungen bereits an. Zudem nutzen sie Ge- schäftsberichte eher als Medium, um diese gegenüber externen Stakeholdern zu vermarkten. In der Handels- und Konsumgüterbranche (78 Prozent) sowie der Automobilbranche (76 Prozent) dominiert hingegen der qualitative Teil im Vergleich zum Durchschnitt. Die beiden Branchen könnten demnach im Betrachtungszeitraum am stärksten von der Digitalisierung betroffen gewesen sein oder sich mitten in der Transformation befinden.

\section{Schlussbetrachtung}

Die Ergebnisse der Geschäftsberichtsanalyse zeigen, dass sich der Digitalisierungsgrad der 30 DAX-Unternehmen sowohl nach Bereichen als auch nach Branchen stark unterscheidet. Häufig haben die Konzerne Schwierigkeiten, konkrete Digitalisierungsmaßnahmen bei der Entwicklung neuer Geschäftsmodelle umzusetzen. Unternehmen sollten ihre bisherige Unternehmenssteuerung deshalb überdenken und die

\section{„Unternehmen sollten ihre Unternehmens- steuerung überdenken und Digitalisierung als verbindliches und messbares Element in das Controlling einbetten."}

Digitalisierung als verbindliches und messbares Element in das Controlling einbetten. Steuerungskennzahlen, die den Digitalisierungsgrad unterschiedlicher Handlungsfelder widerspiegeln, können traditionelle Steuerungskennzahlen dabei effektiv ergänzen: Beispielsweise könnten Unternehmen digitale Ziele in bisherige Instrumente zur Leistungsmessung oder Performance-Messung wie in einer Balanced Scorecard integrieren. Auf diese Weise ließen sich die vier Perspektiven Kunden, Finanzen, Prozesse und Entwicklung um eine weitere Perspektive erweitern, welche sich ausschließlich mit den Handlungsfeldern der Digitalisierung befasst, beispielsweise mit digitalen Geschäftsmodellen oder Prozessen. Alternativ lassen sich digitale Ziele auch innerhalb der bereits bestehenden Perspektiven einbetten. Der digitale Zustand eines Unternehmens kann mittels verschiedener Reifegradmodelle aus Literatur und Praxis ermittelt werden.

Um digitale Aktivitäten über alle Ebenen hinweg konsequent umsetzen und ganzheitlich steuern zu können, müssen Unternehmen zudem ihr Reporting anpassen. Der Fortschritt digitaler Projekte, aber auch der Ergebnis- und Fixkostenbeitrag der entsprechenden Initiativen sollten deshalb auf der 
Agenda der monatlichen Berichterstattung stehen. Auch lassen sich digitale Vorhaben in die individuelle Zielvereinbarung integrieren, um digitale Aktivitäten zu fördern. Einige wenige DAX-Konzerne wie Daimler haben digitale Ziele wie beispielsweise den Anteil von digitalem Self Service oder Umsatz an Mobilitätsdienstleistung bereits in der individuellen Zielvereinbarung auf Vorstandsebene verankert.

\section{Literatur}

Mayring, P. (2015): Qualitative Inhaltsanalyse. Grundlagen und Techniken, 12. Auflage, Weinheim.

\section{Angaben zu den Autoren}

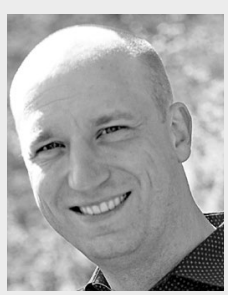

Prof. Dr. Kai Kruk

ist Professor für digitale Ökonomie und Studiengangsleiter an der Hochschule

Worms

E-Mail:kruk@hs-worms.de

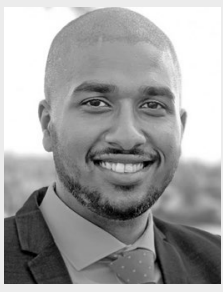

\section{Tennyson Rajenthiran}

ist Consultant im Bereich Risk Advisory bei der Wirtschaftsberatung Deloitte,

Mannheim

E-Mail: terajenthiran@deloitte.de

\section{SpringerGabler}

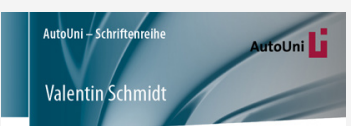

Performancemessung schlanker Produktionssysteme

V. Schmidt

Performancemessung schlanker

Produktionssysteme

2020. XXVIII, 250 S. 47 Abb. Brosch. $€$ (D) $39,99 \mid €$ (A) $41,11 \mid{ }^{*} \mathrm{CHF} 44.50$ ISBN 978-3-658-31020-2

$€$ (D) $29,99 \mid{ }^{*} \mathrm{CHF} 35.50$

ISBN 978-3-658-31021-9 (eBook)

- Modelltheoretische Untersuchung mit mehreren Szenarien

$€(D)$ : gebundener Ladenpreis in Deutschland, $€(A)$ : in Österreich. *: unverbindliche Preisempfehlung. Alle Preise inkl. gesetzl. MwSt.

Schönbohm, A./Egle, U. (2021): Controlling der digitalen Transformation, in: Schallmo, D. R. A. et al. (Hrsg.): Digitale Transformation von Geschäftsmodellen, Wiesbaden, S. 199-223. www.springerprofessional.de/link/19151972

Thaler, M./Pierer von Esch, S. (2020): Planung in Zeiten digitaler Geschäftsmodelle, in: Controlling \& Management Review, 64 (3), S. 8-15. www.springerprofessional.de/link/17908884
Part of SPRINGER NATURE

springer.com/empfehlung 